\title{
Trends in Wait Time for Outpatient Colonoscopy in the Veterans Health Administration, 2008-2015
}

\author{
Megan A. Adams, MD, JD, MSC 1,2,3, Joel H. Rubenstein, MD, MSC ${ }^{1,2,3}$, \\ Rachel Lipson, $M S^{7}$, Robert G. Holleman, $\mathrm{MPH}^{7}$, and Sameer D. Saini, MD, MSc ${ }^{1,2,3}$
}

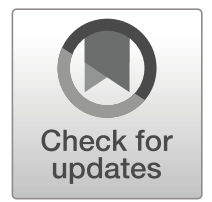

'Center for Clinical Management Research, Department of Veterans Affairs, VA Ann Arbor Healthcare System, Ann Arbor, MI, USA; ${ }^{2}$ Division of Gastroenterology, Department of Internal Medicine, University of Michigan Health System, Ann Arbor, MI, USA; ${ }^{3}$ Institute for Healthcare Policy and Innovation, Ann Arbor, MI, USA.

BACKGROUND: The Veterans Health Administration (VA) recently has been scrutinized for prolonged wait times for routine medical care, including elective outpatient procedures such as colonoscopy. Wait times for colonoscopy following positive fecal occult blood test (FOBT) are associated with worse clinical outcomes only if greater than 6 months. OBJECTIVE: We aimed to investigate time trends in wait time for outpatient colonoscopy in VA and factors influencing wait time.

DESIGN: Retrospective cohort study using mixed-effects regression of VA administrative data from the Corporate Data Warehouse.

PARTICIPANTS: Veterans who underwent outpatient colonoscopy for positive FOBT in 2008-2015 at 124 VA endoscopy facilities.

MAIN MEASURES: The main outcome measure was wait time (in days) between positive FOBT and colonoscopy completion, stratified by year and adjusted for sedation type, year, and potentially influential patient- and facilitylevel factors.

KEY RESULTS: In total, 125,866 outpatient colonoscopy encounters for positive FOBT occurred during the study period. The number of colonoscopies for this indication declined slightly over time $(17,586$ in 2008 vs. 13,245 in 2015; range 13,425-19,814). In 2008, median wait time across sites was 50 days (interquartile range $[\mathrm{IQR}]=33$, 75). There was no secular trend in wait times (2015 median $=52$ days, IQR $=34,77$ ). Examining the adjusted effect of patient- and facility-level factors on wait time, no clinically meaningful difference was found.

CONCLUSIONS: Wait times for colonoscopy for positive FOBT have been stable over time. Despite the perception of prolonged VA wait times, wait times for outpatient colonoscopy for positive FOBT are well below the threshold at which clinically meaningful differences in patient outcomes have been observed.

KEY WORDS: colonoscopy; access; veterans.

J Gen Intern Med 35(6): 1776-82

DOI: $10.1007 / \mathrm{s} 11606-020-05776-4$

(C) Society of General Internal Medicine (This is a U.S. government work and not under copyright protection in the U.S.; foreign copyright protection may apply) 2020

Received August 22, 2019

Revised January 30, 2020

Accepted March 6, 2020

Published online March 24, 2020

\section{INTRODUCTION}

The Veterans Health Administration (VA) has faced increased scrutiny in recent years over timely access to high-quality primary and specialty care services. As a result, ensuring that veterans have timely access to care has become a key policy priority for the VA. ${ }^{1}$ VA has been documenting wait times for each appointment type since 1999, when the Congress first requested information on VA outpatient wait times. ${ }^{2}$ In the wake of highly publicized reports of wait time manipulation and access issues at the Phoenix VA and other sites in 2014, VA began publicly posting wait-time data for new and return visits to VA primary and specialty care clinics nationwide. ${ }^{3,4}$ But little clarity exists regarding the threshold of wait time that represents an appropriate versus inappropriate or harmful delay in care. A common way to assess appropriateness is by comparing wait times between delivery systems in the same healthcare market, but this approach primarily serves to inform timeliness from a consumer satisfaction perspective rather than a medical appropriateness perspective. As VA undergoes an unprecedented expansion of VA Community Care, as legislatively mandated through the VA Maintaining Internal Systems and Strengthening Integrated Outside Networks (MISSION) Act of 2018, how the VA is able to optimize timely access for VA-enrolled veterans in its own facilities will have significant implications on the extent to which veterans' care will be outsourced to community settings. Indeed, supply-demand mismatch is a structural challenge for the VA and many other healthcare systems which can be difficult to address.

In this study, we aimed to investigate time trends in wait time for outpatient colonoscopy in the VA performed for an indication of a positive fecal occult blood test (FOBT). We limited our analysis to colonoscopies performed for this indication because it enabled us to pinpoint an objective time point when waiting commenced for an elective procedure with a clearly specified indication. There are also excellent data available regarding how long of a delay in colonoscopy after positive FOBT is associated with worse clinical outcomes (> 6 months). ${ }^{5}{ }^{6}$ We hypothesized that, contrary to public perception, overall wait times for colonoscopy have remained steady or decreased over time in VA reflecting expansion of 
clinical resources in the face of a growing population of VAenrolled veterans. ${ }^{7}$ Furthermore, we hypothesized that VA wait times would be within a clinically acceptable range. We also aimed to better understand factors associated with increased wait time, including the effect of endoscopic sedation type. In previous studies, we demonstrated significant increases in the use of anesthesia assistance (AA) for routine GI endoscopy in VA facilities, particularly since $2011 .{ }^{8,}{ }^{9}$ We hypothesized that median wait time for colonoscopy with AA would be clinically significantly longer than wait time for patients referred for colonoscopy with standard sedation, given fewer available anesthesia appointments.

\section{METHODS}

This study was approved by the Ann Arbor VA IRB. This was a retrospective study using VA administrative data obtained through the VA Corporate Data Warehouse.

\section{Study Population}

The study population consisted of veterans who underwent outpatient colonoscopy in 2008-2015 for an indication of positive FOBT. We focused on those with positive FOBT in order to define an objective time point when waiting commenced. Cases were identified via Current Procedural Terminology (CPT) codes for colonoscopy, cross-referenced with laboratory data to identify patients with a positive FOBT in the 6 months leading up to the procedure. ${ }^{10}$ Sensitivity analysis was also performed using a 12-month window between positive FOBT and colonoscopy. Cases performed with AA were identified by searching for applicable procedure codes occurring on the same day as anesthesia CPT code 00810 (colonoscopy) or 00740 (upper endoscopy) using a validated algorithm. ${ }^{8}$ The latter CPT code was included to account for the possibility of bidirectional endoscopy with AA coded only with the upper endoscopy. Patients with an ICD-9 code for rectal bleeding $\leq 6$ months prior to FOBT were excluded.

\section{Data Analysis}

Mixed effects regression was used to model the data. The primary outcome was median facility-specific wait time (in days) between positive FOBT and colonoscopy completion. A cube root transformation was implemented to improve the model fit, with results reported on the original scale of days. The primary model was adjusted for sedation type and other potentially influential patient- and facility-level confounders. Patient-level factors included age, gender, and Charlson-Deyo comorbidity index (CCI) score. We included patient-level covariates in the analysis since these factors could potentially influence colonoscopy wait times. For instance, patients with increased comorbidity could receive higher priority in scheduling (perhaps related to advocacy by a primary care provider (PCP) on their behalf). Alternatively, such patients could experience longer wait times if increased monitoring/ resources are needed to safely complete their procedure and available slots for enhanced monitoring are limited. The yearspecific proportion of all colonoscopy procedures referred from the veteran's PCP facility using AA, categorized into quartiles of use (based on 2012 data), was included in the analysis as a facility-level factor. AA utilization was included as a facility level, rather than a patient-level factor because AA is a resource-limited service at some VA facilities, and we hypothesized that patients having colonoscopies at facilities with lower overall levels of AA use (potentially reflecting lower access to AA) may experience longer wait times than patients having colonoscopies at facilities with higher overall levels of AA use (reflecting greater access to AA). PCP facility was defined as the site with the most primary care contacts with the veteran over the 2 years preceding FOBT. Patients without VA primary care contacts or assigned to PCP facilities that did not refer any patients for colonoscopy during the study period were excluded. PCP facility was included in the model as a random effect. All statistical analyses were conducted using SAS Version 9.4 (SAS Institute, Cary, NC). We also explored additional facility-level factors, including 2011 VA facility complexity model score (incorporating factors including patient risk, clinical volume, teaching/research activity, and ICU level, rated on a scale from 1a (highest complexity) to 3 (lowest complexity)) and geographic region (West, Plains, Central, Northeast). Facilities with no assigned complexity level $(n=3)$ were designated "unclassified." Results are reported as means and standard deviations, as well as medians and interquartile ranges (IQR). The impact of patient- and facility-level predictors on wait time was examined using predictive marginal analysis with $95 \%$ confidence intervals (CI).

\section{RESULTS}

A total of 125,866 outpatient colonoscopy encounters following positive FOBT occurred during the study period at $124 \mathrm{VA}$ facilities. Of these, $5.3 \%$ (6694 procedures) were performed with AA. Frequencies of patient and facility characteristics are shown in Table 1. Patients in the cohort were predominantly male and generally healthy, with a mean age of 62.9 years. The number of FOBTs followed by colonoscopies remained roughly stable between 2008 and 2011 (17,586 in 2008 vs. 16,183 in 2011) but declined slightly thereafter (range 13,24514,222) (Table 2). The number of VA facilities offering AA increased from 64 in 2008 to 110 in 2015 . The median number of colonoscopies performed per facility for this indication was 1643 (IQR 1044-2420).

\section{Colonoscopy Wait Times}

In 2008, the median wait time for colonoscopy across sites was 50 days (IQR $=33,75)$, and there was no secular trend in wait times $(2015$ median $=52$ days; IQR 34, 77) $($ Table 2). 
Table 1 Demographics

\begin{tabular}{ll}
\hline \hline $\begin{array}{l}\text { Veteran demographics } \\
\text { Gender }\end{array}$ & $\mathbf{N ( \% )}$ \\
Male $(n, \%)$ & $120,499(95.7 \%)$ \\
Female $(n, \%)$ & $5367(4.3 \%)$ \\
Age at FOBT & \\
$\leq 50(n, \%)$ & $7277(5.8 \%)$ \\
$51-65(n, \%)$ & $74,236(59.0 \%)$ \\
$66-75(n, \%)$ & $34,515(27.4 \%)$ \\
$76-85(n, \%)$ & $8936(7.1 \%)$ \\
$\geq 86(n, \%)$ & $902(0.7 \%)$ \\
Charlson comorbidity index score & \\
$0(n, \%)$ & $76,432(60.7 \%)$ \\
$1-2(n, \%)$ & $37,476(29.7 \%)$ \\
$\geq 3(n, \%)$ & $11,958(9.5 \%)$ \\
Facility variables & $\mathbf{N}(\%)$ \\
Colonoscopy facility region & \\
West & $23,931(19.0 \%)$ \\
Plains & $40,757(32.4 \%)$ \\
Central & $49,179(39.1 \%)$ \\
Northeast & $11,999(9.5 \%)$ \\
Colonoscopy facility complexity level & \\
1a & $47,009(37.4 \%)$ \\
1b & $22,903(18.2 \%)$ \\
1c & $23,545(18.7 \%)$ \\
2 & $20,380(16.2 \%)$ \\
3 & $11,647(9.3 \%)$ \\
Unclassified & $382(0.3 \%)$ \\
\hline
\end{tabular}

Across years, there was a maximum difference in median wait time of only 8 days ( 47 days in 2009 vs. 55 days in 2011 and 2012), which was not considered clinically significant. A sensitivity analysis was performed using a 12-month window between positive FOBT and colonoscopy completion (rather than 6 months), and the median wait time for colonoscopy was $\leq 4$ days longer across years. Across years, $9.1 \%$ of patients completed their colonoscopy $>6$ months after positive FOBT (range 8.0-10.8\%), though the reason for the delay could not be discerned from available data. Facility-level variability in wait time between positive FOBT and colonoscopy at all VA endoscopy facilities in FY 2015 is shown in Figure 1. At the facility with the longest median wait time (limited to facilities performing $\geq 10$ colonoscopies in 2015), veterans waited 68 days longer for a colonoscopy than at the facility with the shortest median wait time (94 days vs. 26 days, respectively). At the facility with the longest median wait time, $75 \%$ of colonoscopies were completed within 131 days.

Among patients with a positive FOBT, $45 \%$ had not completed a colonoscopy (either at a VA facility or in the community via fee-basis) at 12 months, and $42 \%$ had not completed a colonoscopy at 24 months after positive FOBT.

Table 2 Trend in Unadjusted Wait Times (in Days) for Outpatient Colonoscopy for Positive FOBT in VA Facilities, 2008-2015

\begin{tabular}{lllllll}
\hline $\begin{array}{l}\text { FOBT } \\
\text { year }\end{array}$ & $\boldsymbol{N}$ & $\begin{array}{l}\text { Median } \\
\text { days }\end{array}$ & $\begin{array}{l}\text { 25th } \\
\text { Pctl }\end{array}$ & $\begin{array}{l}\text { 75th } \\
\text { Pctl }\end{array}$ & Mean & $\begin{array}{l}\text { Std } \\
\text { Dev }\end{array}$ \\
\hline 2008 & 17,586 & 50.00 & 33.00 & 75.00 & 59.79 & 37.20 \\
2009 & 17,603 & 47.00 & 31.00 & 68.00 & 55.84 & 35.27 \\
2010 & 19,814 & 52.00 & 35.00 & 78.00 & 61.40 & 37.01 \\
2011 & 16,183 & 55.00 & 35.00 & 84.00 & 64.68 & 39.55 \\
2012 & 13,455 & 55.00 & 37.00 & 84.00 & 64.46 & 38.46 \\
2013 & 13,758 & 50.00 & 34.00 & 76.00 & 59.92 & 36.94 \\
2014 & 14,222 & 49.00 & 33.00 & 74.00 & 58.22 & 36.31 \\
2015 & 13,245 & 52.00 & 34.00 & 77.00 & 60.19 & 36.59 \\
\hline
\end{tabular}

This percentage is consistent with previous studies of CRC screening in VA. ${ }^{10,11}$

\section{Effect of Sedation Type on Colonoscopy Wait Times}

Examining the adjusted effect of endoscopic sedation type on wait time trends (Table 3 ), no clinically meaningful difference was found. The difference in adjusted mean wait times for colonoscopies with standard sedation vs. AA was found to be $\leq 4$ days across years.

\section{Marginal Effects of Covariates on Wait Time}

The marginal effects of all patient- and facility-level predictors on wait time are presented in Table 4. None of the examined predictors was found to influence colonoscopy wait time in any clinically meaningful way. The year of the procedure influenced wait time by $\leq 4$ days from the reference year (2008). A similar magnitude of effect was seen between primary care facilities with minimal AA use and those with higher usage. Wait times by patient age group differed by 1.4 days or less. Patients with higher $\mathrm{CCI}$ scores $(\mathrm{CCI} \geq 3$ ) experienced slightly longer wait times for colonoscopy than healthy patients (CCI 0 ), but the difference was $<3$ days. Again, this is not a clinically meaningful difference for this indication. VA facilities located in the Northeast had the shortest wait times, while facilities in the Central region had the longest wait times (difference of 6.4 days). Mean wait time at the highest (level 1a) and lowest (level 3) complexity facilities differed by only 7 days.

\section{DISCUSSION}

Improving veterans' access to specialty care has been an ongoing focus of VA and many other healthcare systems, as they seek to address supply-demand mismatch in system-wide healthcare delivery. Gastroenterology is one of the most referred-to specialties by PCPs, ${ }^{12}$ in large part related to gastroenterologists' role in colorectal cancer (CRC) screening. CRC screening both within and outside VA is largely done directly through colonoscopy, but referral for colonoscopy may also be triggered by a positive FOBT, an accepted primary screening modality. In this study, we sought to better characterize access to colonoscopy in VA over an 8-year period by examining procedural wait times. We limited our analysis to colonoscopies performed for an indication of a positive FOBT because it enabled us to pinpoint an objective time point when waiting commenced for an elective procedure with a clearly specified indication. Consistent with our hypothesis, we found no clinically meaningful increase in wait time among patients who completed an outpatient colonoscopy for this indication across the study period. Median wait time across years was approximately 50 days ( $<2$ months) between positive FOBT and colonoscopy. Applying a 12- 


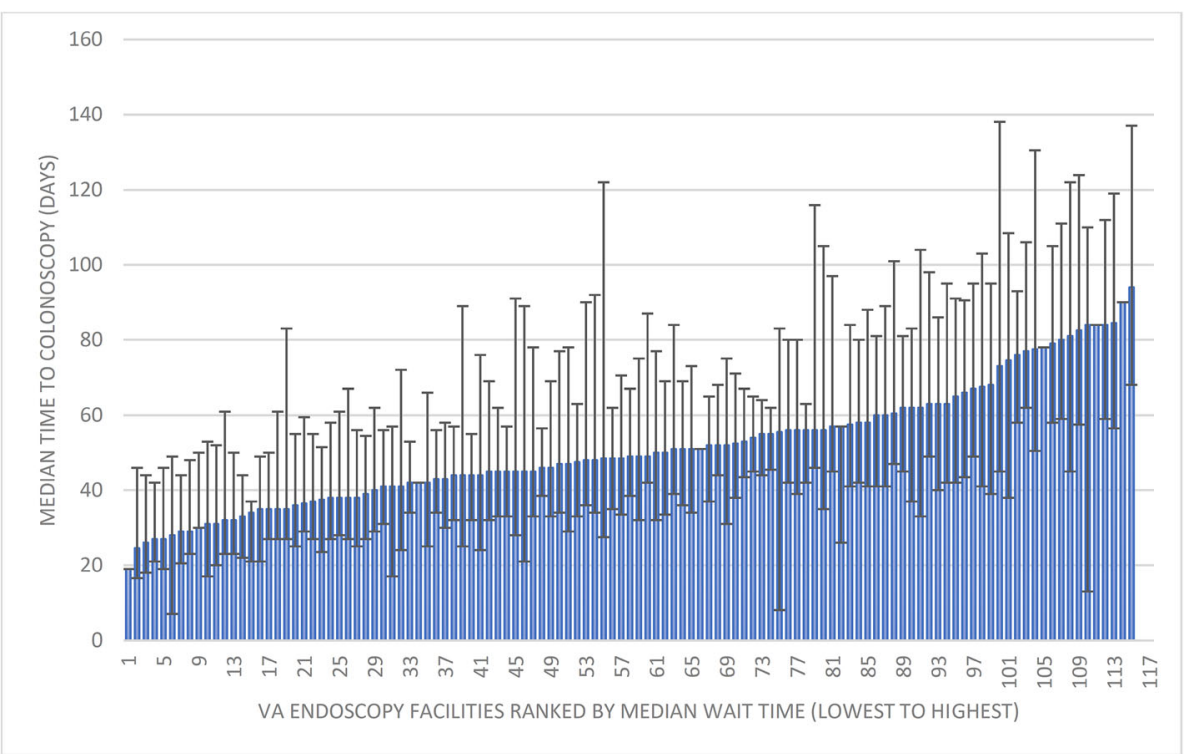

Figure 1 VA endoscopy facilities ranked by median wait time (in days), lowest to highest, between positive FOBT and colonoscopy completion in fiscal year 2015.

month time window between FOBT and colonoscopy completion in sensitivity analysis (rather than 6 months) had no meaningful impact on median wait time (maximum difference of 4 days across years). Notably, approximately $9 \%$ of veterans completed their colonoscopy $>6$ months after positive FOBT, a potentially concerning finding given data on worse outcomes for individuals who wait $>6$ months for colonoscopy after positive FOBT. However, many potential reasons other than a delay in initial scheduling/access may explain this phenomenon, including patient factors such as canceling and rescheduling the procedure or the lack of driver availability leading to deferral of scheduling. Moreover, we can expect that some small proportion of individuals will not undergo colonoscopy at the recommended interval despite efforts to ensure that they do so. For example, approximately 3\% of patients in Kaiser Permanente Northern/Southern California
(2010-2014) experienced a > 6-month delay between positive FOBT and colonoscopy when using a similar 12-month window. ${ }^{5}$ While this number is slightly lower than that observed in our study, the patient populations served by VA and Kaiser are different, and VA faces unique challenges related to not only its complex patient population but also to the geographic dispersion of its population. Thus, this variation may be explained by the differences in patient population, regional differences, or reflect true differences in patient access. Identifying the reasons for these delays in colonoscopy completion among outliers is an important area for future study. Finally, it is important to acknowledge the prior body of work conducted by VA on this topic, including systematic quality improvement efforts to improve appropriate and timely follow-up after positive FOBT. Our data suggest that these efforts, including the VA CRC Care Collaborative, have continued to yield

Table 3 Trend in Mean Adjusted Wait Times for Outpatient Colonoscopy for Positive FOBT in VA Facilities, by Year and Type of Endoscopic Sedation

\begin{tabular}{|c|c|c|c|c|c|}
\hline Year & $\begin{array}{l}\text { Sedation type } \\
\text { (SS, standard sedation; } \\
\text { AA, anesthesia-assistance) }\end{array}$ & $\begin{array}{l}\text { Number of colonoscopy } \\
\text { encounters }\end{array}$ & $\begin{array}{l}\text { Mean wait } \\
\text { time (days) }\end{array}$ & $\begin{array}{l}\text { Lower confidence } \\
\text { interval (days) }\end{array}$ & $\begin{array}{l}\text { Upper confidence } \\
\text { interval (days) }\end{array}$ \\
\hline \multirow[t]{2}{*}{2008} & SS & 17,210 & 50.7 & 48.7 & 52.8 \\
\hline & AA & 376 & 54.6 & 52.3 & 57.0 \\
\hline \multirow[t]{2}{*}{2009} & SS & 17,237 & 47.4 & 45.5 & 49.4 \\
\hline & AA & 366 & 51.1 & 48.9 & 53.4 \\
\hline \multirow[t]{2}{*}{2010} & SS & 19,448 & 51.1 & 49.1 & 53.2 \\
\hline & AA & 366 & 55.0 & 52.7 & 57.4 \\
\hline \multirow[t]{2}{*}{2011} & SS & 15,472 & 53.7 & 51.5 & 55.8 \\
\hline & AA & 711 & 57.7 & 55.3 & 60.2 \\
\hline \multirow[t]{2}{*}{2012} & SS & 12,735 & 54.0 & 51.9 & 56.2 \\
\hline & AA & 720 & 58.1 & 55.7 & 60.6 \\
\hline \multirow[t]{2}{*}{2013} & SS & 12,687 & 49.7 & 47.7 & 51.7 \\
\hline & AA & 1071 & 53.5 & 51.2 & 55.9 \\
\hline \multirow[t]{2}{*}{2014} & SS & 12,904 & 47.5 & 45.6 & 49.5 \\
\hline & AA & 1318 & 51.3 & 49.0 & 53.6 \\
\hline \multirow[t]{2}{*}{2015} & SS & 11,479 & 49.1 & 47.1 & 51.2 \\
\hline & AA & 1766 & 52.9 & 50.7 & 55.3 \\
\hline
\end{tabular}


Table 4 Adjusted Analysis of the Impact of Patient- and FacilityLevel Factors on Wait Time

\begin{tabular}{|c|c|c|}
\hline Predictor & $\begin{array}{l}\text { Wait time (in days), adjusting } \\
\text { for other covariates (margin } \\
\text { with } 95 \% \text { CI) }\end{array}$ & $\begin{array}{l}\text { Wait time } \\
\text { difference (in } \\
\text { days) }\end{array}$ \\
\hline \multicolumn{3}{|l|}{ Year } \\
\hline 2008 & $52.7(50.5-54.8)$ & Reference \\
\hline 2009 & $49.3(47.2-51.4)$ & -3.4 \\
\hline 2010 & $53(50.9-55.2)$ & 0.3 \\
\hline 2011 & $55.7(53.5-57.9)$ & 3.0 \\
\hline 2012 & $56.1(53.8-58.3)$ & 3.4 \\
\hline 2013 & $51.6(49.5-53.7)$ & -1.1 \\
\hline 2014 & $49.4(47.3-51.5)$ & -3.3 \\
\hline 2015 & $51(48.9-53.2)$ & -1.7 \\
\hline \multicolumn{3}{|l|}{ Age } \\
\hline$\leq 50$ & $51.7(49.6-53.9)$ & Reference \\
\hline$\overline{5} 1-65$ & $53.1(51.1-55.2)$ & 1.4 \\
\hline $66-75$ & $52.8(50.7-54.9)$ & 1.1 \\
\hline $76-85$ & $52.6(50.5-54.8)$ & 0.9 \\
\hline$\geq 86$ & $51.3(48.5-54.1)$ & -0.4 \\
\hline \multicolumn{3}{|c|}{ 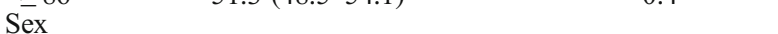 } \\
\hline Male & $52.4(50.2-54.7)$ & Reference \\
\hline Female & $52.2(50.1-54.2)$ & -0.3 \\
\hline \multicolumn{3}{|c|}{ Charlson comorbidity index score } \\
\hline 0 & $51(49-53.1)$ & Reference \\
\hline $1-2$ & $52.2(50.1-54.3)$ & 1.2 \\
\hline$>3$ & $53.7(51.5-55.9)$ & 2.7 \\
\hline \multicolumn{3}{|c|}{$\mathrm{PC} \bar{P}$ facility quartiles of AA use for all colonoscopies } \\
\hline Zero AA use & $50.4(52.5-50.4)$ & Reference \\
\hline Q1 & $49.9(52-49.9)$ & -0.5 \\
\hline Q2 & $51.4(53.5-51.4)$ & 1.0 \\
\hline Q3 & $54.9(57.1-54.9)$ & 4.5 \\
\hline Q4 & $55.1(57.4-55.1)$ & 4.7 \\
\hline \multicolumn{3}{|c|}{ Colonoscopy facility region } \\
\hline West & $54.3(51.7-57)$ & Reference \\
\hline Plains & $52.6(50.2-55)$ & -1.7 \\
\hline Central & $54.6(52.2-57)$ & 0.3 \\
\hline Northeast & $47.9(45.2-50.6)$ & -6.4 \\
\hline \multicolumn{3}{|c|}{ Colonoscopy facility complexity level } \\
\hline $1 \mathrm{a}$ & $56.4(54.3-58.6)$ & Reference \\
\hline $1 \mathrm{~b}$ & $56.1(53.7-58.6)$ & -0.3 \\
\hline $1 \mathrm{c}$ & $53.9(51.7-56.3)$ & -2.5 \\
\hline 2 & $52.2(50.1-54.4)$ & -4.2 \\
\hline 3 & $49.3(47-51.6)$ & -7.1 \\
\hline Unclassified & $46.3(40.5-52.6)$ & -10.1 \\
\hline
\end{tabular}

improvements over time. ${ }^{13}$ While a significant proportion of veterans $(42 \%)$ did not have a colonoscopy within 2 years of positive FOBT, this likely represents a ceiling estimate given the dual enrollment of some veterans in Medicare and other programs.

Campaigns to reduce patient wait times for medical procedures and other care are predicated on the assumption that longer wait times lead to poorer health outcomes. In a recent retrospective cohort study of 70,124 Kaiser Permanente Northern California patients who underwent colonoscopy for a positive fecal immunochemical test (FIT), Corley et al. found no increased risk of CRC or advanced-stage disease associated with colonoscopy follow-up within 6 months, compared with 8-30 days, but increased risk of poor outcomes if colonoscopy was performed $>6$ months from the positive FIT. $^{5}$ A subsequent study of 39,326 patients participating in Taiwan's Nationwide CRC Screening Program found a statistically significant difference in the risk of CRC and advancedstage disease when colonoscopy was delayed $>6$ months from positive FOBT, compared with 1-3 months, but not with waits of 4-5 months. ${ }^{6}$ Viewed through this lens, VA colonoscopy wait times are well within a clinically acceptable range based on available evidence. But there is often a disconnect between delays in care that may be justifiable based on medical evidence (i.e., scheduling a colonoscopy 5 months, rather than 2 weeks, after positive FOBT) and what is appropriate from a patient or health system perspective. Indeed, there is typically a great deal of patient anxiety upon receipt of a positive cancer screening test, emotions that are often best addressed with prompt scheduling and completion of follow-up testing. Not surprisingly, studies have shown that longer wait times not only are associated with lower overall patient satisfaction with care but also affect patient perceptions of their providers and quality of care. ${ }^{14}$ Furthermore, PCPs and health system administrators unfamiliar with this evidence on clinical outcomes may similarly feel a sense of urgency and seek to address delays in care that are otherwise clinically appropriate.

Various factors, beyond the simple availability of endoscopy slots, may influence colonoscopy wait time. However, in our analysis, potentially influential patient- and facility-level factors examined did not influence overall colonoscopy wait time in any clinically meaningful way. Availability of services ancillary to endoscopy, such as specialized sedation, theoretically may further delay care if supply is inadequate to meet demand. However, we found no clinically meaningful difference in procedural wait times for patients referred for colonoscopy with AA versus standard sedation. This may be a consequence, in part, of referral of patients requiring $\mathrm{AA}$ to community facilities (through VA purchased care programs) if wait times would be prolonged within VA. Furthermore, delays in referral for colonoscopy after positive FOBT (due to provider delay or difficulties contacting the patient) can lead to longer wait times irrespective of procedural capacity. Also, patient reticence to undergo the procedure and/or cancelation and rescheduling of the procedure may result in an artificial delay on the review of administrative data. While the examination of wait times in administrative data has its limitations, it does offer an important window into large-scale trends in healthcare delivery and timeliness of care.

Our study has several limitations that deserve mention, including the inherent risk of misclassification using administrative data. However, we previously validated CPT codes for detection of sedation type, ${ }^{8}$ and the use of CPT codes for colonoscopy has also been previously validated in VA. ${ }^{15}$ Furthermore, our study results may not be generalizable to all colonoscopy indications, as we limited our analysis to a single colonoscopy indication in order to accurately specify the time when waiting commenced. VA has made systematic efforts to improve the timeliness of colonoscopy after positive FOBT. ${ }^{12}$ Additionally, as outlined above, we did not examine the effect of outsourced care on VA facility wait times for colonoscopy. This is due to challenges in accessing reliable national non-VA data in this area. However, available evidence suggests that wait times for colonoscopy do not vary 
substantially between veterans receiving colonoscopy in VA versus non-VA settings, at least at the regional level. ${ }^{16}$ Finally, in focusing our study on colonoscopies completed within 6 months of a positive FOBT, we may have missed patients who had a colonoscopy $>180$ days after a positive FOBT for this indication. However, as the time between positive FOBT and colonoscopy completion elapses, it becomes increasingly difficult to discern whether the patient had the colonoscopy for an indication of positive FOBT or for some other indication that arose in the intervening period (e.g., diarrhea, imaging abnormality). Additionally, the distribution of wait times (Fig. 1) indicates that very few veterans even approached waiting 180 days for colonoscopy, such that it is unlikely that large numbers of colonoscopies for this indication were excluded. Finally, our sensitivity analysis showed that median wait time for colonoscopy was no greater than 4 days longer across years when a 12-month time window between positive FOBT and colonoscopy was utilized.

Our study adds to the existing literature in several notable ways. First, to our knowledge, it represents the first (nonoperational) attempt to quantify wait times in the VA nationally using a meaningful wait time metric (time between positive FOBT and colonoscopy completion). Second, no prior study has assessed the effect of endoscopic sedation type on access to colonoscopy within or outside VA. Given frequent anecdotal reports by VA gastroenterologists that patients experience delays in scheduling for colonoscopies with AA, the fact that wait times for colonoscopies with AA observed in this study were not clinically significant was surprising. However, this could reflect the outsourcing of patients requiring AA for endoscopic procedures to non-VA settings when wait times are prolonged.

\section{CONCLUSION}

Wait times for colonoscopy in the VA remained steady between 2008 and 2015, despite an expanding population of VA-enrolled veterans, reflecting expansion of clinical resources. While waiting $<2$ months for colonoscopy after positive FOBT appears unlikely to result in an increased risk of CRC or advanced-stage disease based on available evidence, continued efforts to expand supply and moderate demand through the more appropriate use of endoscopic resources are warranted in order to shorten wait times and optimize overall patient satisfaction and perceptions of care quality.

Specific Author Contributions: Megan A. Adams, MD, JD, MSc: study concept and design, acquisition of data, analysis and interpretation of data, statistical analysis, and drafting the manuscript. Joel H. Rubenstein, MD, MSc: study concept and design, analysis and interpretation of data, and critical revision of the manuscript for important intellectual content.
Rachel Lipson, MSc: acquisition, analysis, and interpretation of data. Robert G. Holleman, MPH: acquisition, analysis, and interpretation of data.

Sameer D. Saini, MD, MSc: study concept and design, analysis and interpretation of data, critical revision of the manuscript for important intellectual content, and study supervision.

Each author has approved the final draft submitted.

Corresponding Author: Megan A. Adams, MD, JD, MSc; Division of Gastroenterology, Department of Internal Medicine University of Michigan Health System, Ann Arbor, MI, USA (e-mail: meganada@med. umich.edu).

Funding Information This study was supported by the Veterans Health Administration's Office of Informatics and Analytics. The opinions are those of the authors and do not represent those of the Department of Veterans Affairs. Megan A. Adams was supported by an American College of Gastroenterology Junior Faculty Development Grant (2018-2021) during the preparation of this manuscript.

\section{Compliance with Ethical Standards:}

Guarantor of the Article: Megan A. Adams.

Conflict of Interest: The authors declare that they do not have a conflict of interest.

Disclaimer: The Veterans Health Administration had no role in the design, conduct, or analysis of this study or the decision to submit the manuscript for publication.

\section{REFERENCES}

1. U.S. Department of Veterans Affairs FY 2018-2024 Strategic Plan. Available at: https://www.va.gov/oei/docs/va2018-2024strategicplan. pdf. Accessed 1/16/19.

2. U.S. General Accounting Office. VA needs better data on extent and causes of waiting times; 2000. GAO/HEHS-00-90).

3. Shulkin D. Understanding Veteran wait times. Ann Intern Med 2017;167(1):52-54.

4. U.S. Department of Veterans Affairs. Average wait times at individual facilities. Available at: https://www.va.gov/oei/docs/va2018-2024strategicplan.pdf. Accessed 1/16/19.

5. Corley DA, Jensen CD, Guinn VP, et al. Association between time to colonoscopy after a positive fecal test result and risk of colorectal cancer and cancer stage at diagnosis. JAMA 2017;317(16):1631-1641.

6. Lee YC, Fann JC, Chiang TH. Time to colonoscopy and risk of colorectal cancer in patients with positive results from fecal immunochemical tests. Clin Gastroenterol Hepatol (In press)

7. Congressional Research Service. The number of Veterans that use VA health care services: a fact sheet. June 3, 2014. Available at: https://fas. org/sgp/crs/misc/R43579.pdf. Accessed 1/8/19.

8. Adams MA, Prenovost KM, Dominitz JA, Holleman RG, Kerr EA Krein SL, Saini SD, Rubenstein JH. Predictors of utilization of monitored anesthesia care for outpatient gastrointestinal endoscopy in a capitated payment system. Gastroenterology 2017;153:1496-1503.

9. Adams MA, Prenovost KM, Dominitz JA, Kerr EA, Krein SL, Saini SD, Rubenstein $\mathbf{J H}$. National trends in utilization of monitored anesthesia care for outpatient gastrointestinal endoscopy in the Veterans Health Administration. JAMA Intern Med 2017;177:436-438.

10. Partin MR, Gravely AA, Burgess JF JR, et al. Contribution of patient, physician and environmental factors to demographic and health variation in colonoscopy follow-up for abnormal colorectal cancer screening test results. Cancer 2017; 123:3502-3512.

11. Carlson CM, Kirby KA, Casadei MA, et al. Lack of follow-up after fecal occult blood testing in older adults: inappropriate screening or failure to follow up? Arch Intern Med. 2011;171:249-256).

12. Barnett ML, Song $\mathbf{Z}$, Landon BE. Trends in physician referrals in the United States, 1999-2009. Arch Intern Med 2012;172(2):163-170. 
13. Powell AA, Nugent S, Ordin DL et al., Evaluation of a VHA collaborative to improve follow-up after a positive colorectal cancer screening test. Med Care 2011;49(10):897-903.

14. Bleustein C, Rothschild DB, Valen A, et al. Wait times, patient satisfaction scores, and the perception of care. Am J Manag Care 2014;20(5):393-400.

15. Fisher DA, Grubber JM, Castor JM, Coffman CJ. Ascertainment of colonoscopy indication using administrative data. Dig Dis Sci 2010;55(6):1721-1725.
16. May FP, Yano BM, Provenzale D, et al. Barriers to follow-up colonoscopies for patients with positive results from fecal immunochemical tests during colorectal cancer screening. Clin Gastroenterol Hepatol 2019;17(3):469-476.

Publisher's Note: Springer Nature remains neutral with regard to jurisdictional claims in published maps and institutional affiliations. 